\title{
VOTING MULTIDIMENSIONAL DATA AND SELECTING BEST SERVICE PROVIDER USING QOS
}

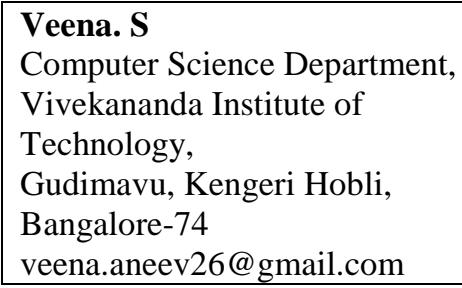

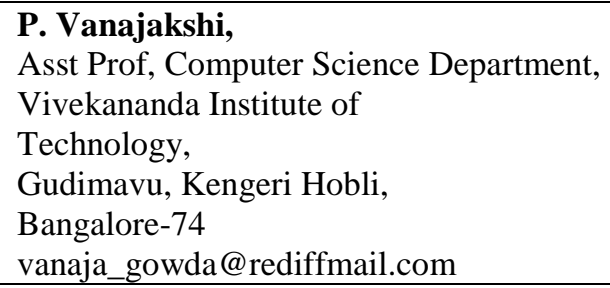

\begin{abstract}
Cloud computing is a technology which provides computing resources on demand and is a solution for utility computing. There are many cloud service providers, as the number of cloud service provider increases which are having similar functionalities then it is a problem for cloud users for the selection of cloud service providers. To overcome with this problem we have assisted the users as per their requirements during their selection of cloud service providers. User may provide its QoS expectation and the offers will be expressed by service providers. Experience of existing users may be very useful for selecting the best clod service provider. It identifies QoS metrics and defines it in such a way that user can express their expectation and provider can express their offers into quantified form. Hence a dynamic and flexible framework using Ranked Voting Method is proposed which takes the input as user requirement and provides the output as the best cloud service provider.
\end{abstract}

Keywords - Cloud Computing; Service Measure Index; Ranked Voting; Quality of Service.

\section{INTRODUCTION}

Cloud computing, emerged as a latest example for utility computing, and is rapidly growing. It has gained the attention in not only large organizations but also in government organizations, even in academic organizations. Cloud computing and utility, both offers for delivering on-demand resources. Cloud computing provides three types of services: SaaS, PaaS and IaaS three deployment models: Public, Private and Hybrid Cloud.

Big and academic organizations and even users can use the benefits provided by cloud computing. Cloud users have to pay only for the operational cost, where in the Traditional data centre reduces the computing cost significantly. Customer can rent more computing resources from Cloud when the company is growing without bothering to pay for unneeded resources. Organization becomes more agile as the cloud customers need not bother about the maintenance of infrastructure.

Many service providers offer same service on different costs with vary in performance than other service providers. Some providers charge high and some low for CPU and RAM respectively. Increasing number of service providers is making more competitive in cloud marketing day by day. Each service provider claims their best, which makes the customers difficult for selecting a provider which fulfils users $\mathrm{QoS}$ requirement.

For selecting best cloud service provider a user must identify its QoS measures which is used to compare with the various service providers. QoS measurement is difficult to understand as there are a lot of standards to measure QoS. A framework called SMI which is a standard measurement was developed by CSMIC. There are 7 characteristics, where there are several attributes for each characteristic. A framework has been designed which considers not only metrics defined by SMI but also others metric found in analysis of QoS parameters. There are 2 types of metrics: user dependent and application dependent metrics. To rank the providers, we used ranked voting method. Each metric will 
behave as a voter and it compares its metric value with the provider's value for that particular metric. After getting ranked voting data which contains voter list, ranked voting method was used which was proposed by Tsuneshi Obata and Hiroaki [1] which is used to find the best cloud service provider.

\section{RELATED WORK}

\section{A Method for Discriminating Efficient Candidates With Ranked Voting}

Ranked voting data arise when voters select and rank more than one candidate with order of preference. Cook and Kress introduced DEA to analyzed ranked voting data. By using DEA, each candidate may use the most favorable weights for them to estimate their own score of their preference. However DEA often suggests that more than one unit are equally efficient. So a method to discriminate these efficient candidates is needed. Some methods of such purpose are proposed. However, these methods have a weak point that the order of efficient candidates may be changed by existence of an inefficient candidate. Our method does not use information about inefficient candidates. It is based on a very simple idea.

Cloud computing Landscape and Research challenges Regarding Trust and Reputation

Cloud computing is an emerging computing paradigm. It shares scalable and elastic resources (eg data, calculations and services) transparently among the users over a massive network. The cloud market is growing very rapidly and bringing up numerous research challenges. It provides a landscape of cloud computing and its research and challenges, especially considering the areas of e selecting the service, quality assurance of cloud services and trust establishment in cloud environments.

\section{SMI (SERVICE MEASURE INDEX)}

The service measure index is currently being developed by Cloud Services Measurement Initiative Consortium (CSMIC) [2]. It consists of business-relevant Key Performance Indicators (KPI) that provide a standardization method for measuring and comparing a business service. The SMI framework provides a entire view of QoS needed by the customers for selecting a Cloud service provider based on: Accountability, Agility, Assurance of Service, Financial, Performance, Security and Privacy, and Usability. There are many attributes in each category. Each attributes helps to define the metric in detail and also helps in collecting data to measure that particular metric. Although all attributes in list are important, this work provides flexibility to user to select some attributes as voter according to its need.

There are currently no publicly available metrics or methods which define these KPIs and compare Cloud providers. SMI is the first effort. Figure 1 shows the hierarchy of SMI framework. The attributes definitions provided here are all written using these basic terms.

Service: A set of requirements or functions made available to a group of users by service provider.

User: The people that interact directly with a service.

Client: An organization that acquires one or more services for its users.

Service Provider: An structured group that provides one or more services to one or more clients.

Service Level Agreement: An licensed union between a service provider and a client documenting quantitative service level commitments. 
ELK

Asia Pacific Journals

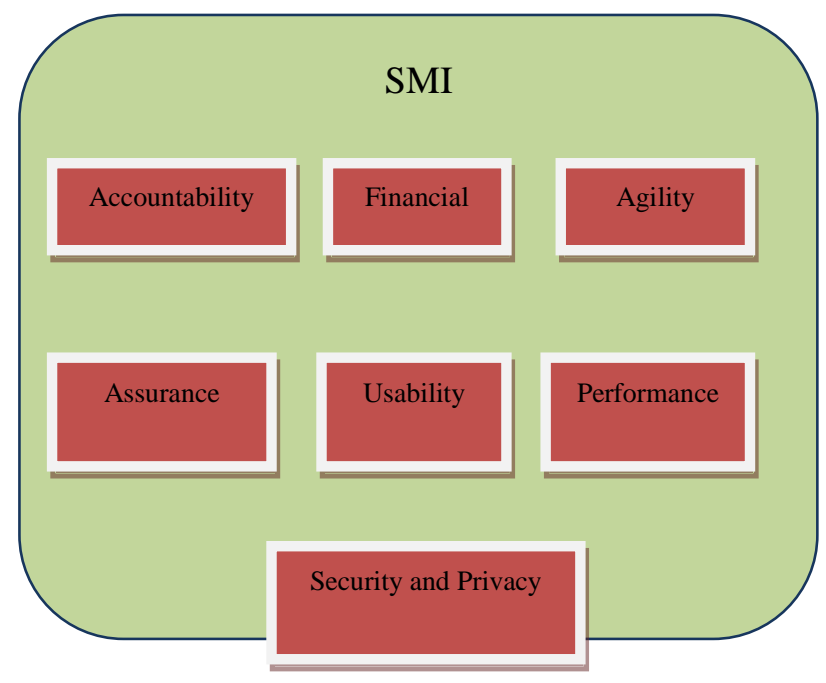

Figure 1: Categories of SMI

Accountability- This group of QoS attributes is used to measure various Cloud provider specific characteristics. It is important to build the trust of a customer on any Cloud provider.

Agility- It is the impact of a service upon client's ability to change direction, strategy, or tactics quickly and with minimal disruption.

Financial- The total cash spent by client.

Performance - Solutions offered by Cloud providers are different addressing the IT needs of different organizations.

Assurance- This characteristic indicates the likelihood of a Cloud service performing as expected or promised in the SLA. Every organization provide better services to their customers.

Security and Privacy- Data protection and privacy are important concerns for nearly every organization. Usability- For the rapid adoption of Cloud services, it plays an significant role.

\section{GLOBAL VIEW OF CLOUD COMPUTING}

There are many numbers of service providers in Cloud market. They advertise their services in their own way. Experience of users also plays an important role in choosing a best provider.
ELK Asia Pacific Journals - Special Issue ISBN: 978-81-930411-5-4

Architecture is given below which brings together all Cloud providers which makes implementation of proposed framework easy. A global view of Cloud computing as a Federated Cloud or Inter Cloud [3] has been proposed. Figure 2 depicts such Cloud architecture.

The components of Cloud architecture are shown below.

\section{A. Cloud Exchange}

Cloud exchange acts as central coordinator which binds together the Cloud providers, Cloud coordinator, Cloud user and Cloud broker. It contains directory to provide information to broker about Cloud services.

\section{B. Directory}

Directory contains information which is required for selection of a best service provider. It contains Service catalogue and Service info.

\section{Service Catalogue}

All service providers give the details about the services on the basis of defined metric.

\section{Service Info}

It is a repository which has Service log and Review $\log$. Service $\log$ is a database which contains log records of all registered providers. Log records stores all the information about history of service providers. Review log has information about experience of user for a particular service.

\section{Cloud Broker}

Cloud broker has the details of requirement of user and details of service provider from directory and analyse them using proposed framework in order to select the best provider. Essential QoS is informed for the broker by the user, if that QoS is not offered by a service provider, provider will be rejected by the broker for comparison. Cloud broker directly interact with users, it collects information about their experience from users for used service providers, and update service info when required.

D. Cloud Coordinator 
ELK

Asia Pacific Journals

Cloud coordinator acts as a representative for Cloud service provider. It is responsible to provide service catalogue and service $\log$ and periodically update both.

\section{QOS METRICS}

There are 2 types of metrics: Application dependent and user dependent. Application dependent metrics are defined based on the application's requirement and user Dependent metrics are defined on user's requirement basis.

\section{A. Application Dependent Metrics}

Availability

It is the percentage of time a customer can access the service.

Reliability

It reflects how a service operates without failure during a given time and condition.

Response Time

It is the difference between time of request for the service and time when service is available.

Operating Systems Support

Providers support different OS like Mac OS X, Windows, and Open SUSE Linux etc. It is possible that one provider support some OS and other provider supports some other OS like Windows Azure supports only Windows operating system,

while GoGrid supports Windows server 2003/2008.

Platforms Supported

Service Providers can support different OS namely Mac OS X,Windows, and Open SUSE Linux. One Provider may support some OS and the other provider may support other OS.

Throughput and efficiency

Throughput and efficiency are important measures to evaluate the performance of infrastructure services provided by Clouds. Throughput is the number of assigned work completed by the Cloud service per unit of time Cost

Cost is used to rank providers. Cost is a function of requirement of resources like CPU, memory,
ELK Asia Pacific Journals - Special Issue

ISBN: 978-81-930411-5-4

storage in an application. There are two types of pricing plan they are: Price bundling and Unbundling [4].

Capacity

It is a high amount of resources that a provider can provide to users at peak time.

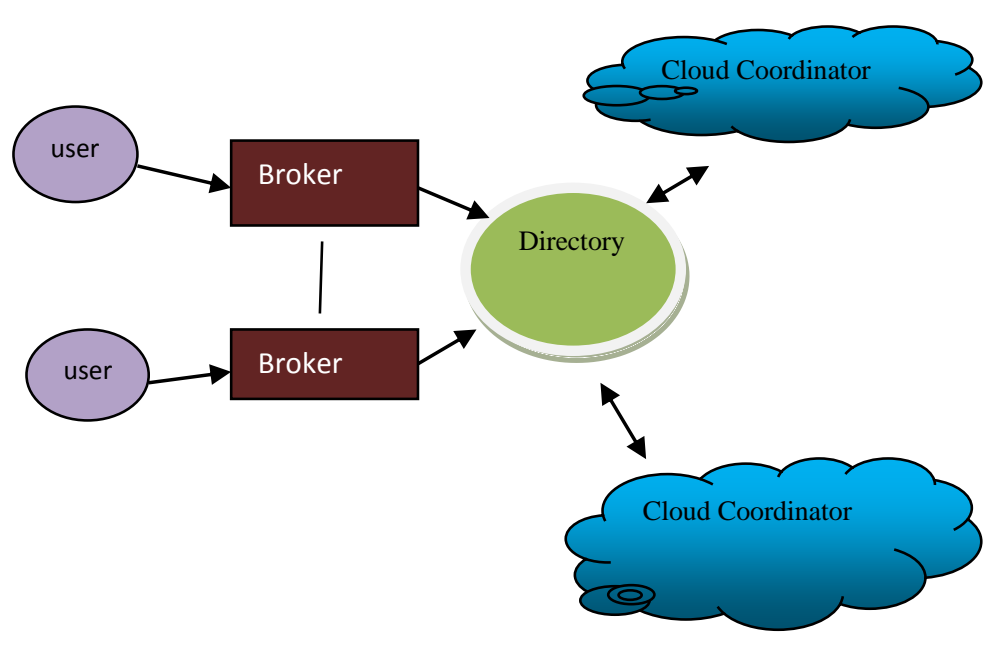

Figure 2: Global View of Cloud Computing

\section{B User Dependent Metrics}

Reputation

It measures trustworthiness of a Cloud service provider. Service providers takes the experience of users.

Sustainability

Sustainability is defined in terms of the environmental impact of the Cloud service used. It can be measured as the energy efficiency of the Cloud service. The property of Sustainability is divided as Accountability attribute, which is used to measure properties related to the service provider organization itself independent of services being provided.

Scalability

Scalability is evaluated in order to determine whether a system can handle a large number of application requests simultaneously or not. The 
scalability has two dimensions: horizontal Cloud scalability ('scale out') and vertical Cloud scalability ('scale up'). Horizontal Cloud scalability means increasing Cloud resources of the same types such as initiating more virtual machines of the same type during peak load. Vertical scalability is an important quality measure for organizations who want to move to the Cloud.

Elasticity

Elasticity is defined in terms of how much a Cloud service can be scaled during prime times. This is defined by two attributes: mean time taken to expand or contract the service capacity, and maximum capacity of service. The maximum number of measure units that can be provided at peak times is the capacity.

Free Trial

To test their services some Providers provide free trial. It is very beneficial for users. User can test services before deployment.

API

Whether provider is providing an API to interact with server or not is also an important parameter.

User Experience

User, takes the services from Cloud, must consider the views of existing users for the services. Existing users of a service can give brief detail about stability and transparency of the service in a better way.

\section{RANKING OF CLOUD SERVICE PROVIDERS}

Each metrics will act as a voter, Cloud providers are candidates for them. Some methods have been proposed to analyse ranked voting data such as Data Envelopment Analysis (DEA) introduced by Cook and Kress. But DEA suggests more than one efficient candidate. Thus Ranked voting method, was introduced by Tsuneshi Obata and Hiroaki Ishii. The numbers of Cloud providers in market must be less than the best Cloud providers that a voter has to select them and rank from top to bottom.

\section{METRIC COMPARISION RULES}

Metric may have different values like numerical value can be provided to cost, Boolean value can be provided to Scalability etc, which can help voters to rank service providers in order of their preference.

\section{NUMERIC}

Numeric value can be given to a metric if the metric value of service provider is large as possible,example Availability

Numeric value can also be given to a metric if the metric value of service provider is as low as possible, example. Cost

\section{BOOLEAN}

Boolean type has two values yes or no. Provider with positive response can have its value as yes or with rank one. Provider with negative response can have its value as no or with rank two.

\section{RESULT AND ANALYSIS}

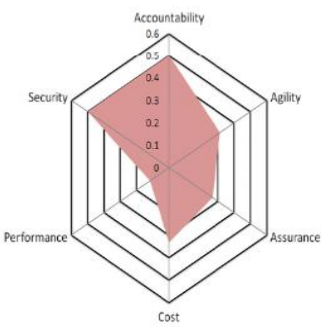

3(a)

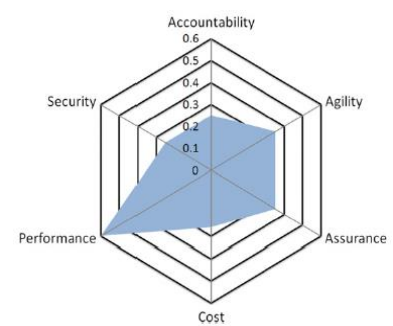

3(b)
FIGURE 3(a). Cloud Service S1 (b). Cloud Service S2

In terms of performance of the machine, Cloud service $\mathrm{S} 2$ is best, however it is low in terms of security. Therefore, S2 is a good alternative for the scientific community where lower priority requirement is security and data is available publicly and it is also the most expensive. On the other side, Cloud service S1 is the good in terms of security which is the key requirement for a user. 
ELK

Asia Pacific Journals

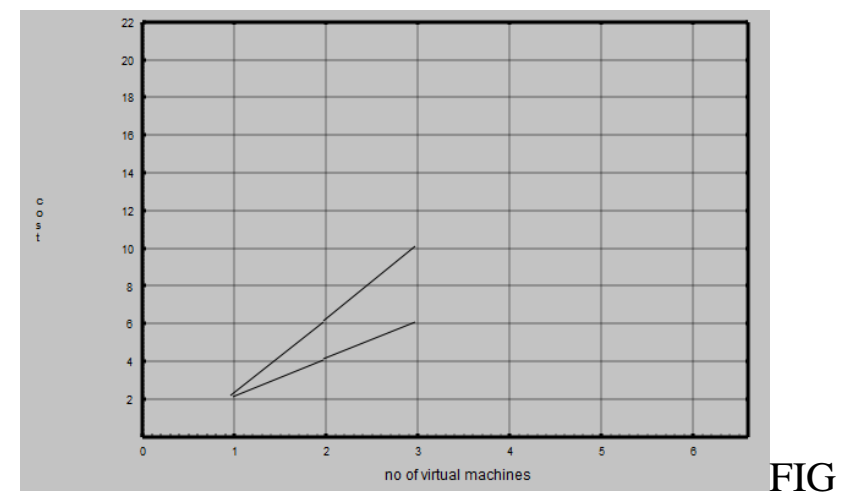

URE 4: Comparison of cost without using algorithm and with using algorithm

The graph shows that the comparison of cost with virtual machines without using algorithm versus with using algorithm. The cost of the machine is high when used without algorithm and the cost is low when used with algorithm.

\section{CONCLUSION AND FUTURE WORK}

Cloud computing provides computing resources on demand and resources are offered on pay-per use basis. With a credit card any one can take services from cloud and deploy and configure servers. So more users are taking services from Cloud which is leading to the growth of cloud market which also in turn is increasing the number of service providers. Increasing number of providers has created confusion in selection of an service provider. Hence QoS metrics are defined to measure the Cloud services and a framework is proposed for selecting best provider using Ranked Voting Method.

User can make their own voter list as their requirement. A set of rules is made to compare QoS values even though they may be of different value types. User may group more than one application to run on a single service provider or user may run applications on different service providers, the proposed framework suggests a best provider. Future work will concentrate on the implementation part to study the
ELK Asia Pacific Journals - Special Issue ISBN: 978-81-930411-5-4

performance of the proposed framework and check in the user reviews who have used the services the keywords are extracted from the review to give the new user an efficient cloud service provider.

\section{REFERENCES}

[1] T. Obata, H. Ishii, "A method for discriminating efficient candidates with ranked voting," European Journal of Operational Research.

[2] J. Siegel, J. Perdue, "Cloud services measures for global use: the Service Measurement Index (SMI).

[3] R. Buyya, C. S. Yeo, S. Venugopal, J. Broberg, I. Brandic, "Cloud Computing and Emerging IT Platforms: Vision, Hype, and Reality for Delivering Computing as the 5th Utility",

[4] S. E. Kihal,C. Schlereth, B. Skiera, "Price Comparison for Infrastructure as a Service," in European Conference on Information Systems, Barcelona, Spain, 2012.

[5] J. Karlsson, K. Ryan, A cost-value approach for prioritizing requirements, IEEE Software.

[6] Armbrust M, Fox A, Griffith R, Joseph $\mathrm{AD}$, Katz R, Konwinski A, Lee G, Patterson D, Rabkin A, Stoica I, Zaharia M (2010) A view of cloud computing.

[7] http://www.cloudorado.com/. 